\title{
A MICROMECHANICAL APPROACH TO NUMERICAL MODELING OF YIELDING OF OPEN-CELL POROUS STRUCTURES UNDER COMPRESSIVE LOADS
}

\author{
REZA HeDAyati \\ Amirkabir University of Technology (Tehran Polytechnic), Department of Mechanical Engineering, Hafez Ave, Tehran, Iran \\ Delft University of Technology (TU Delft), Department of Biomechanical Engineering, Delft, The Netherlands \\ e-mail:rezahedayati@gmail.com; r.hedayati@tudelft.nl; rezahedayati@aut.ac.ir \\ Mojtaba SAdighi \\ Amirkabir University of Technology (Tehran Polytechnic), Department of Mechanical Engineering, Hafez Ave, Tehran
}

Today, interconnected open-cell porous structures made of titanium and its alloys are replacing the prevalent solid metals used in bone substitute implants. The advent of additive manufacturing techniques has enabled manufacturing of open-cell structures with arbitrary micro-structural geometry. In this paper, rhombic dodecahedron structures manufactured using SLM technique and tested by Amin Yavari et al. (2014) are investigated numerically using ANSYS and LS-DYNA finite element codes for the modeling of the elastic and postyielding behavior of the lattice structure, respectively. Implementing a micro-mechanical approach to the numerical modeling of the yielding behavior of open-cell porous materials is the main contribution of this work. One of the advantages of micro-mechanical modeling of an open-cell structure is that, in contrast to the macro-mechanical finite element modeling, it is not necessary to obtain several material constants for different foam material models through heavy experimental tests. The results of the study showed that considering the irregularity in defining the cross-sections of the struts decreases both the yielding stress and densification strain of the numerical structure to the values obtained from the experimental tests. Moreover, the stress-strain curve of the irregular structure was much smoother in two points of yielding and densification, which is also observable in experimental plots. Considering the irregularity in the structure also decreased the elastic modulus of the lattice structure by about $20-30 \%$. The post-densification modulus was more influenced by irregularity as it was decreased by more than $50 \%$. In summary, it was demonstrated that using beam elements with variable cross-sections for constructing open-cell biomaterials could result in numerical results sufficiently close to the experimental data.

Keywords: rhombic dodecahedron, additive manufacturing, micro-structure, porous biomaterials, implant, finite element

\section{Introduction}

For many decades, pure Ti and its alloys have been widely used for the manufacturing of metal implants because of their exceptionally good corrosion resistance and excellent biocompatibility. Although the durability of Ti-based biomaterials is quite good, some implants still fail as a result of instability and aseptic loosening. The first cause of aseptic loosening is the huge difference between the stiffness of cortical bone and the metallic biomaterial, which unloads the natural bone and leads to its absorption after a while (a phenomenon known as stress shielding). Introduction of porosity into bulk metals significantly decreases their stiffness to values close to those of the bone which is very advantageous in avoiding stress shielding. The second reason of implant loosening is weak interfacial bond between the implant surface and the living tissue (Campoli 
et al., 2013). The presence of ample spaces inside porous biomaterials allows for extensive body fluid transport through the porous scaffold matrix which triggers bone ingrowth (Head et al., 1995; Lu et al., 1999; Ryan et al., 2006).

Several manufacturing techniques are used for production of porous metals (Ryan et al., 2006) including the space holder technology (Kwok et al., 2008) and additive manufacturing (AM) techniques (Heinl et al., 2008). The main advantage of additive manufacturing techniques as compared to other traditional manufacturing techniques is their ability to manufacture interconnected porous biomaterials with pre-determined unit cell types. Selective laser melting (SLM) (Mullen et al., 2009) and selective electron beam melting (SEBM) (Heinl et al., 2008) are among the AM methods that are used for production of porous metallic biomaterials (Campoli et al., 2013).

There is not a large class of unit cells which after being packed together can create tessellated structures. Cube (Gibson and Ashby, 1997; Luxner et al., 2009; Parthasarathy et al., 2010), truncated cube (Hedayati et al., 2016d), rhombic dodecahedron (Babaee et al., 2012; Borlefis, 2012; Campoli et al., 2013; Hedayati et al., 2016a; Shulmeister et al., 1998), truncated cuboctahedron (Hedayati et al., 2016c), tetrakaidecahedrons (Warren and Kraynik, 1997; Zheng et al., 2014), rhombicuboctahedron (Hedayati et al., 2016e), 3D-Kagome (Ptochos and Labeas, 2012a,b), Pyramidal (Ptochos and Labeas, 2012a), diamond (Ahmadi et al., 2014; Campoli et al., 2013; Hedayati et al., 2016b), truncated octahedron, and Weaire-Phelan (Bitsche et al., 2005; Buffel et al., 2014; Kraynik and Reinelt, 1996) are the main morphologies which have been more extensively studied. The two latter morphologies have also been proposed as the closest morphologies for foams manufactured using traditional foam manufacturing techniques. The rhombic dodecahedron structure has been shown to be a proper unit cell geometry for being used in bone substitute implants. Analytical relationships for elastic properties of this type of structure can be found in (Babaee et al., 2012) and its complete experimental stress-strain curve data can be found in (Amin Yavari et al., 2013).

Although increased porosity and pore size are obviously preferential for new bone growth in Ti implant (Bram et al., 2000; Karageorgiou and Kaplan, 2005), it should be kept in mind that another consequence of the porosity and pore size increase is the reduction of the implant mechanical properties. This reduction is preferential in elastic modulus but undesirable for fatigue durability and yield strength. Thus depending on the intended application, a balance between different mechanical properties and biological performance should be found (Chen et al., 2009).

Analytical, experimental, and numerical methods have been used by different authors for investigating the behavior of 3D open-cell porous structures in static loadings. Analytical relationships look very helpful in predicting the elastic modulus and yield stress of porous structures. Analytical relationships give the user the elastic properties of a specific porous material with particular micro-geometry, pore size, or relative density. However, analytical relationships cannot be easily derived for the post yielding behavior of these structures because after yielding, many complex deformation regimes, such as buckling, plasticity, and contact between adjacent cells are activated simultaneously. For the post-yielding regime, experimental and numerical techniques show to be helpful. Using numerical techniques, such as finite element, is less costly and time-consuming than experimental procedures and also provides the researcher with ability to reconstruct any morphology, strut size, or sample size.

While the macro-mechanical finite element method has been extensively used in prediction of behavior of open-cell porous structures for almost two last decades, the micro-mechanical approach has not been used sufficiently in previous studies. The authors could not find any previous study regarding the numerical modeling of post-yielding behavior of open-cell porous structures by using finite element models made of beam elements. The micro-mechanical modeling approach enables the researchers to observe the mechanisms occurring in the micro-structural scale and helps them find out what main deformation mechanisms are dominating. 
Porous structures (including open-cell structures), when crushed under compression, usually show a three-stage stress-strain curve. In the first stage, similar to most engineering materials, these materials show an elastic linear part. The second stage starts when some parts of cell edges in the open-cell porous structure (or "cell walls" in a closed-cell porous structure) are yielded, and the stress-strain curve becomes non-linear. Since after being yielded, the cell edges are free to move because of the voids in their periphery, the displacement of the sample accumulates greatly(usually for more than 50-60\%) by keeping the applied load constant or by increasing it very slightly. This stage is known as the plateau stage and the (almost constant) stress in this stage is called the plateau stress. When the sample is crushed enough and the neighbor cell edges come into contact with each other, the third stage (densification stage) is started. Since the cell edges are not as free as in the second stage, the load again starts to increase (nonlinearly). The stress and strain at the beginning of the densification stage are called the densification stress and strain, respectively. An appropriate micro-mechanical finite element model for open-cell foams must be capable of predicting all the noted values with acceptable accuracy.

In this paper, the pre- and post-yielding regimes of porous structures manufactured and experimentally tested by Amin Yavari et al. (2013) will be investigated numerically using ANSYS and LS-DYNA finite element packages. In their work, rhombic dodecahedron interconnected cells were created using SLM method. The manufacturing technique was not perfect and there were some imperfections in the micro-structure of the porous structures (Fig. 1). In particular, it was noted that some struts were significantly weaker than others (Amin Yavari et al., 2013). In this paper, the procedure of developing a finite element model for simulating the yielding behavior of the rhombic dodecahedron structure is presented. The effect of considering the irregularity in the cross-section area of the struts, as a way of introducing the presence of imperfections in the actual manufactured samples, on the obtained mechanical properties will also be investigated.

(a)

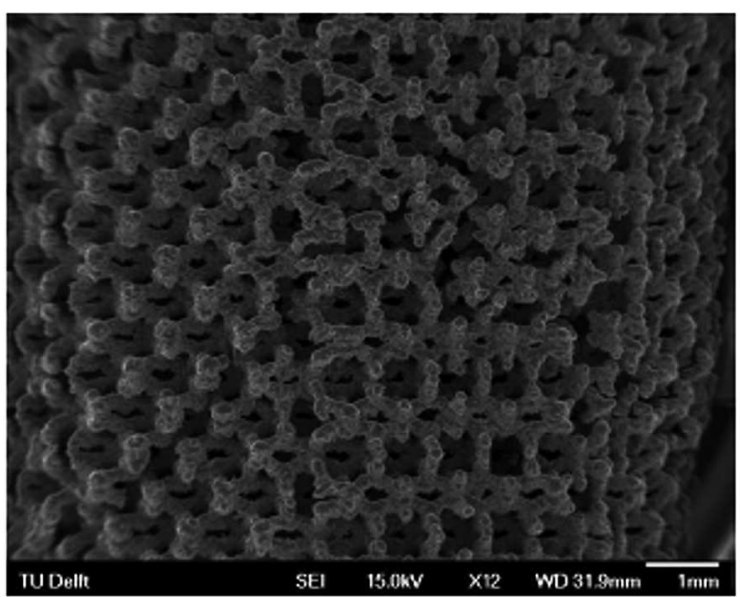

(b)

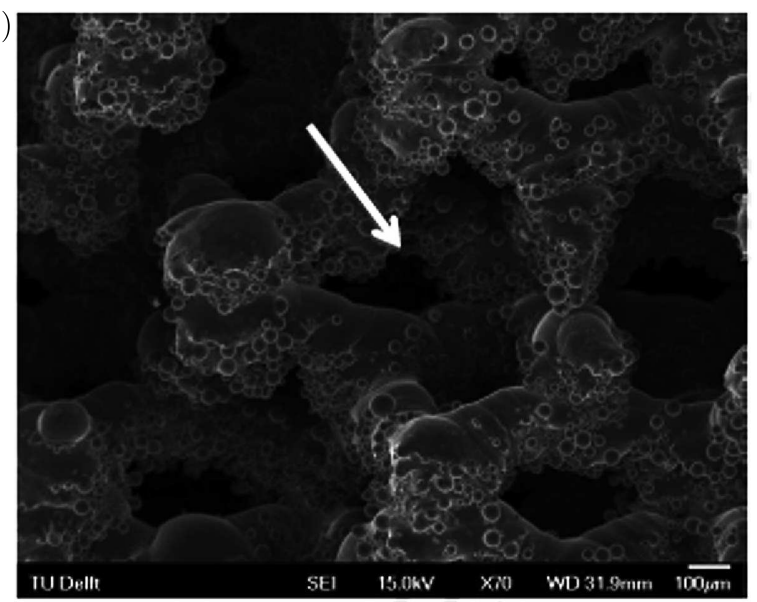

Fig. 1. Micro images of the additively manufactured porous structures (Amin Yavari et al., 2013)

\section{Materials and methods}

\subsection{Numerical modeling}

\subsubsection{Creation of a lattice structure}

If a single unit cell with all of its struts is going to be repeated in the three main directions of space to make a lattice structure, some edges of the neighbor unit cells overlap each other. The overlapping of common edges of the adjacent cells can stiffen the structure in an artificial way leading to incorrect results. To overcome this problem, after creating a unit cell (e.g. cell ABCDEF in Fig. 2), it is checked if it has any edge overlapped on already created edges of 
its adjacent cells. For example in Fig. 2, the newly created edge EF overlies on the previously created edge 23 and edge AF overlies on edge 27. After recognizing the overlapped struts, the edges and nodes attached to each end of the older strut are recognized and their relevant numbers are saved into an array (edges 12,34, 87 and nodes 8, 1 and 4). The older overlapped beam edges (edges 23 and 27) as well as the edges connected to them (edges 12, 34, and 87) are then removed (Fig. 2b). In the next step, the remaining nodes of the removed struts (nodes 1, 4, and 8) are connected to the corresponding nodes of the remaining overlapped strut (edges EF and $\mathrm{AF}$ ) by creating new edge lines (edges 1f, 4E, and A8) (Fig. 2c). While this procedure is robust, it takes a long time for being completed, especially for large 3D structures.
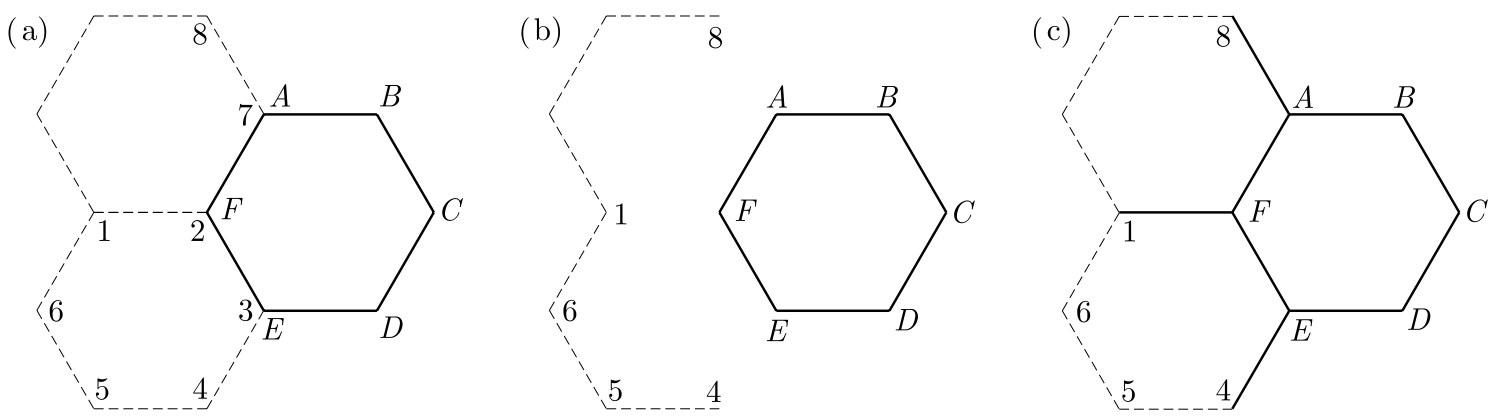

Fig. 2. The procedure of eliminating the overlapping of beam elements

\subsubsection{Modeling the irregularities}

For modelling the elastic behaviour of the specimens, implicit finite element method was used. Four specimens with pore sizes, strut sizes, and porosities listed in Table 1 were simulated. The specimens had relative densities in the range of $68-84 \%$, strut diameters between $140 \mu \mathrm{m}$ and $251 \mu \mathrm{m}$, and pore sizes between $486 \mu \mathrm{m}$ and $608 \mu \mathrm{m}$ (Table 1). Relative density is defined as the ratio of density of the porous structure to density of the bulk material it is made of. The cross-section of the struts had circular cross-sections. The 3D lattice structure of each case was created using Timoshenko beam elements that take into account the shear deformation and rotational inertia effects. Two rigid grips were also modelled and placed under and above the porous structure (Fig. 3).

Table 1. Geometrical specifications of four different samples investigated (Amin Yavari et al., 2013)

\begin{tabular}{|l|c|c|c|c|}
\hline & $\begin{array}{c}\text { Sample 1 } \\
\text { Ti 120-500 }\end{array}$ & $\begin{array}{c}\text { Sample 2 } \\
\text { Ti 170-450 }\end{array}$ & $\begin{array}{c}\text { Sample 3 } \\
\text { Ti 170-500 }\end{array}$ & $\begin{array}{c}\text { Sample 4 } \\
\text { Ti 230-500 }\end{array}$ \\
\hline \hline Dimensions $(D \times L)[\mathrm{mm} \times \mathrm{mm}]$ & $10 \times 15$ & $10 \times 15$ & $10 \times 15$ & $10 \times 15$ \\
\hline Pore size $[\mu \mathrm{m}] \pm \mathrm{SD}$ & $560 \pm 173$ & $486 \pm 162$ & $608 \pm 182$ & $560 \pm 186$ \\
\hline Strut size $[\mu \mathrm{m}] \pm \mathrm{SD}$ & $140 \pm 38$ & $216 \pm 64$ & $218 \pm 62$ & $251 \pm 76$ \\
\hline Porosity $[\%]$ & 84.22 & 71.2 & 77.68 & 68.45 \\
\hline
\end{tabular}

Variations in diameters of the additively manufactured struts were applied to the FE models by discretising the struts by beam elements with different diameters. The diameters of each of the beam elements were taken from a Gaussian distribution function in ANSYS. For the mean and standard deviation of the Gaussian distribution, those of the diameters of the struts of the experimental tests were implemented. Generally, increasing the number of elements along a strut decreases its mechanical properties which leads to a decrease in the overall structure elastic modulus. Especially, it was seen that using more than two beam elements per each strut may lead to instabilities in the yielding analyses. It is because increasing the number of beam 


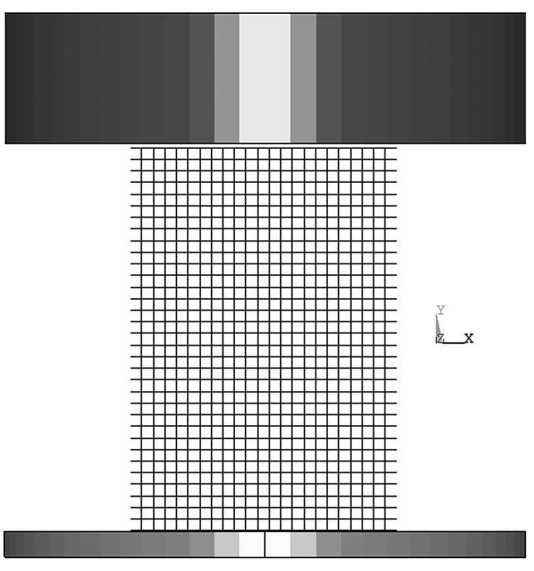

Front view

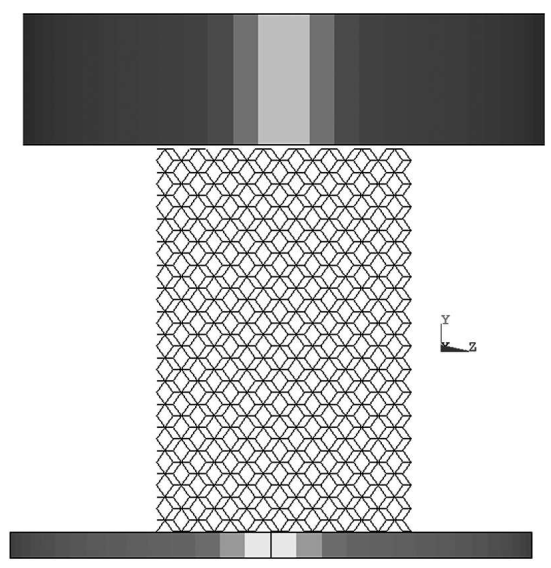

Side view

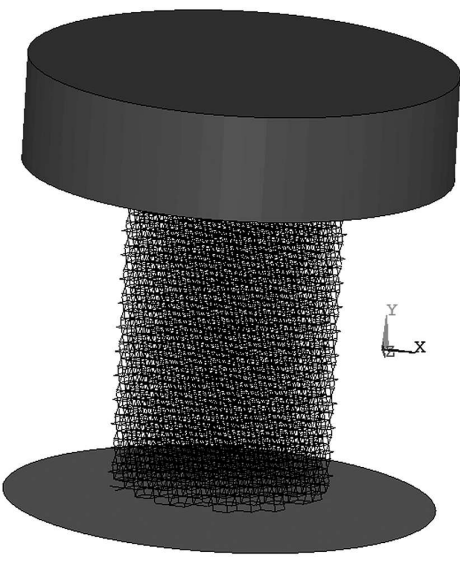

$3 \mathrm{D}$ view

Fig. 3. Finite element model of rhombic dodecahedron lattice structure

elements per strut increases the number of struts with very small cross-sections which will lead to failure of a large group of struts (catastrophic failure). A simple probability calculation can show that the number of weak struts in a three-element-per-strut structure is much higher than a one-element-per-strut structure. To explain this better, consider as an example struts having diameter lower than $d_{c r}$ in a FE lattice structure as weak struts and assume that they constitute $10 \%$ of the FE structure having one element per strut (see Fig. 1b) and $90 \%$ of the struts of this structure are strong. Now the structure with three elements per strut has only $0.9^{3}=72 \%$ strong elements because each strut can be considered strong only if all of its elements have diameters larger than $d_{c r}$. Therefore, the number of weak struts in the three-element-per-struts structure is almost triple of that in the one-element-per-strut structure, which can lead to catastrophic failure of the lattice structure much easier. The considerations taken into account in the Gaussian distribution of our numerical modelling might not also be true in the structures created using the additive manufacturing techniques. For example, it has been seen that in actual SLM-made structures, the majority of weak struts only have one very small cross-section among them (Fig. 1b), while in our numerical modelling, the struts can have more than one weak element. In fact, if it is necessary to use more than one element per strut, more data than just the standard deviation of strut diameter needs to be known.

Another problem that can arise from using more than one element per strut is that most of the beam theories are valid for length/diameter ratios larger than four. For the porous structures considered in this study (Table 1) and in most similar studies, if the number of elements per strut becomes larger than one, then the length/diameter ratio of each beam element becomes smaller than two, which can lead to inaccuracies in obtained stress values for given displacements. Therefore, in all the models created in this study, only one beam element was used to represent each strut. However, diameters of different struts of the FE model were considered to be different. For the FE structure, 21 different strut diameters (ranging between the minimum and maximum diameter values obtained by optical observations) were considered.

\subsubsection{Material model}

For modeling the behavior of the lattice structure in post-yielding regime, an explicit finite element code LS-DYNA was implemented. A plastic kinematic material model (with constants given in Table 2) was used for modeling the mechanical behavior of the metal. In this material model, the material behavior is assumed to be bilinear. Automatic-node-to-surface contact algorithm was used for modeling the interaction between the structure and the grips, and automatic single surface contact algorithm was used for modeling the interactions between neighbor struts. 
The struts were free to move in the direction parallel to the grip faces. The density of $4420 \mathrm{~kg} / \mathrm{m}^{3}$ (Ti6Al4V) was used for the explicit analysis. The lower grip was fixed and the upper grip was moved downward with a constant velocity of $3 \mathrm{~mm} / \mathrm{min}$.

Table 2. Mechanical properties of Ti6Al4V

\begin{tabular}{|l|c|}
\hline \multicolumn{1}{|c|}{ Property } & Value \\
\hline \hline Elastic modulus & $113.8 \mathrm{GPa}$ \\
\hline Poisson's ratio & 0.342 \\
\hline Tangent modulus & $1.25 \mathrm{GPa}$ \\
\hline Yield stress & $1000 \mathrm{MPa}$ \\
\hline Ultimate tensile strength & $1200 \mathrm{MPa}$ \\
\hline
\end{tabular}

\section{Results}

The sequential deformation of the lattice structures having regular and irregular strut cross-section areas are shown in Figs. 4 and 5, respectively. The $45^{\circ}$ failure pattern can be observed in both structures, but more obvious in the regular one (Fig. 4). The failure pattern looks like

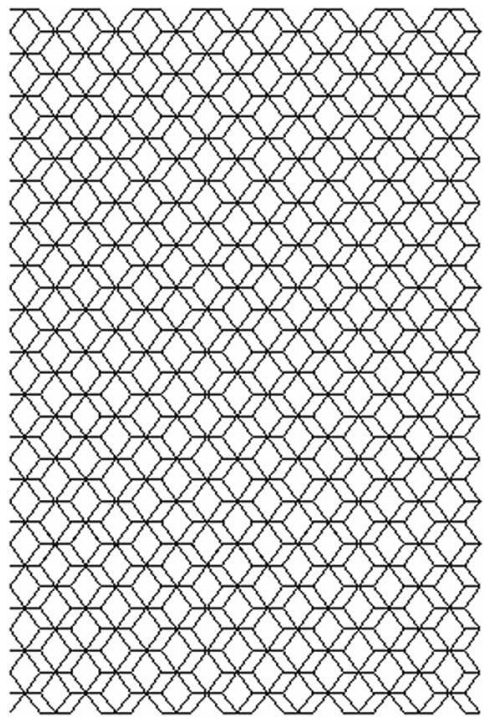

$0 \%$

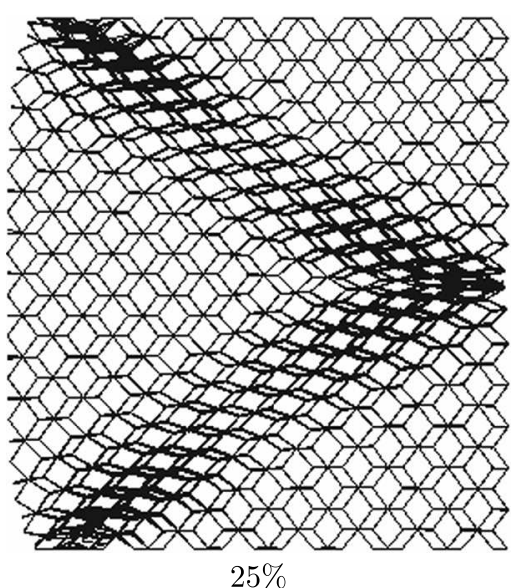

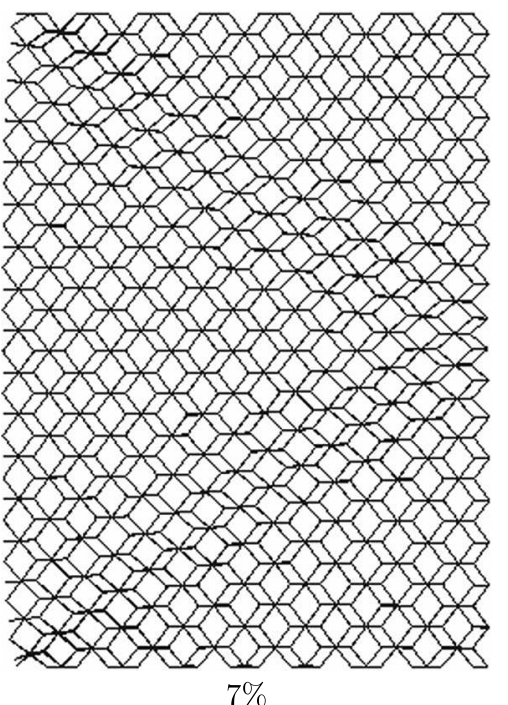
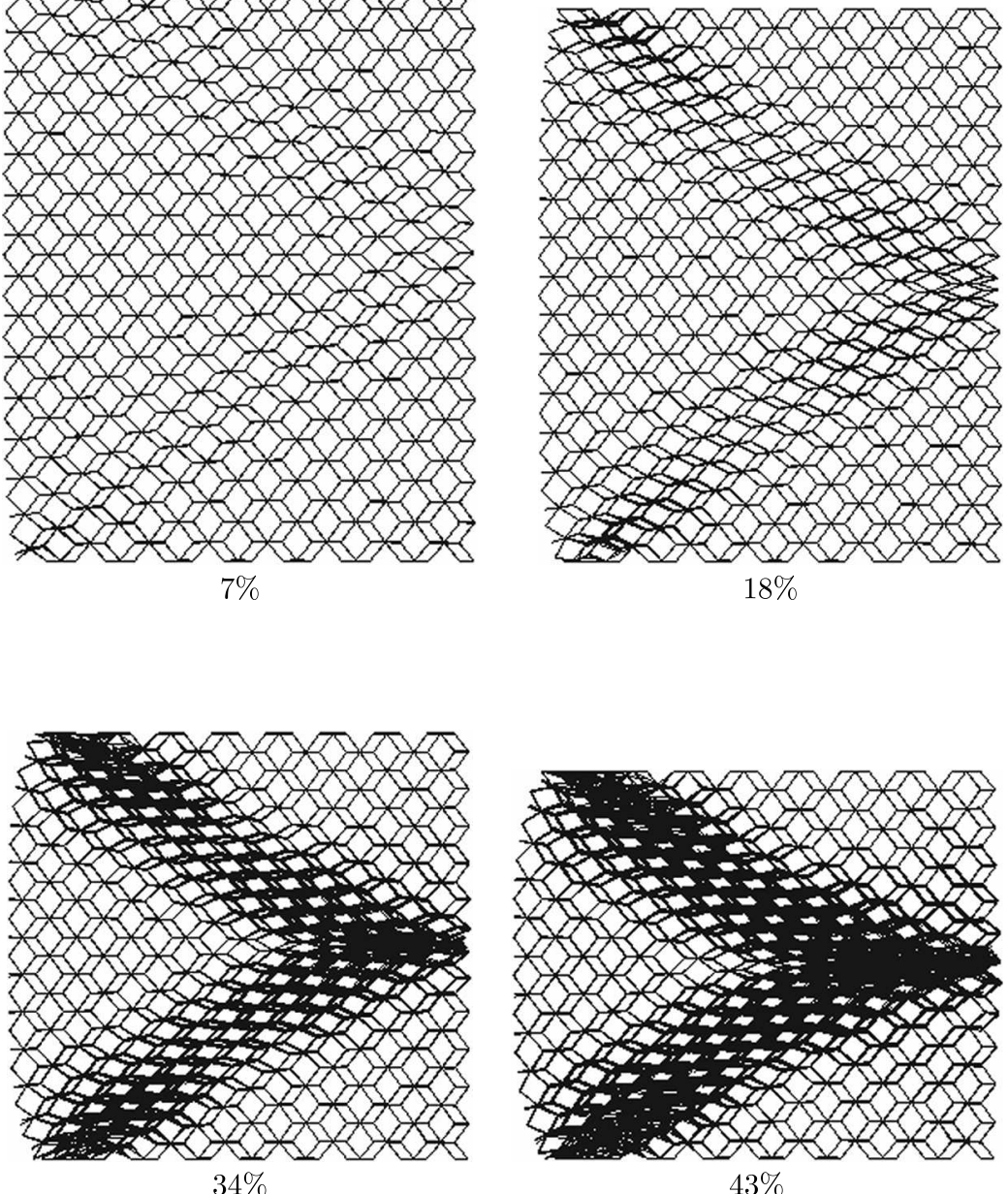

Fig. 4. Deformation of the regular structure at different compressive strains 
a sideways $\mathrm{V}$ in the regular structure, while it is more similar to sideways $\mathrm{X}$ in the irregular structure (Fig. 5). The main deformation mechanisms in the four lattice structures (Table 1) were observed to be first the elastic bending of the struts and then the local plasticity. Local buckling did not occur in the struts. This could be expected because no strut in the initial configuration of this structure was parallel to the direction of loading. The fact that the local yielding occurs far before buckling was also analytically shown for the diamond structure by Ahmadi et al. (2014).

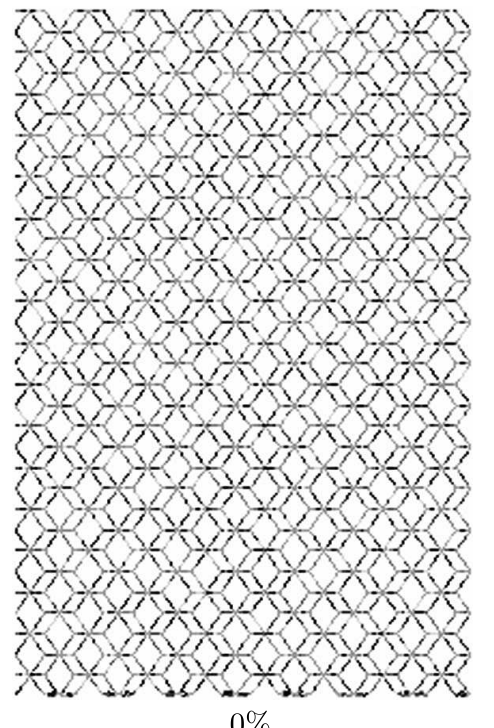

$0 \%$

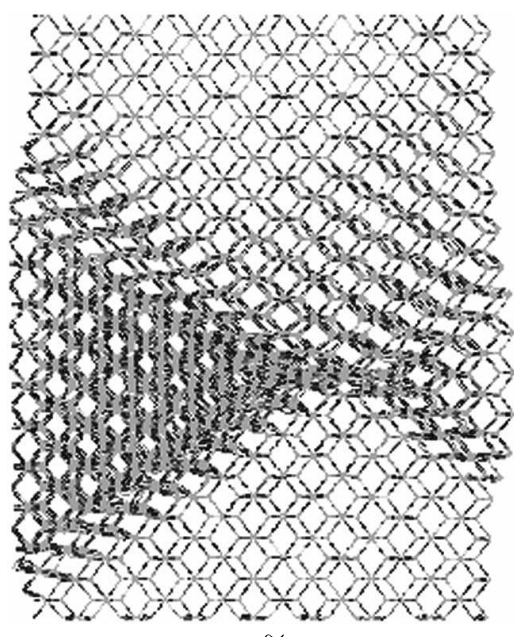

$16 \%$

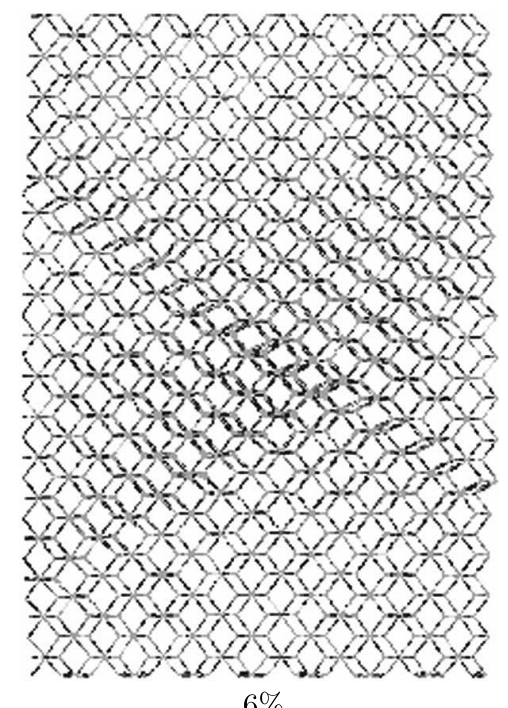

$6 \%$

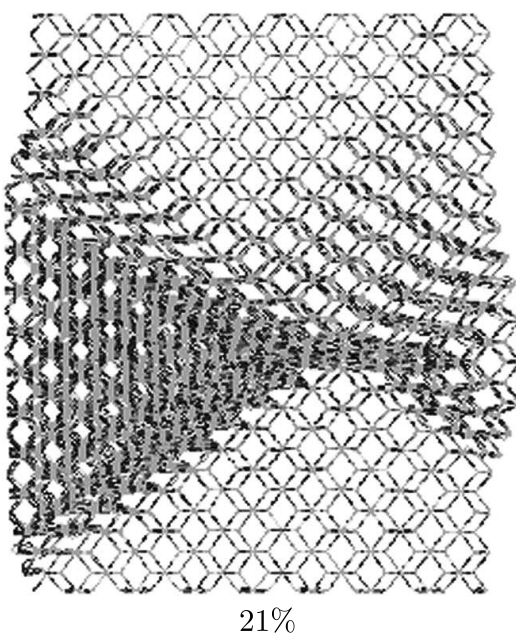

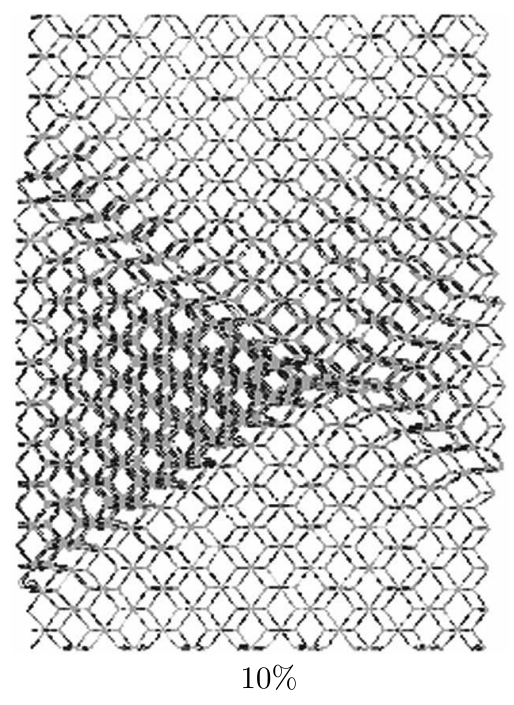

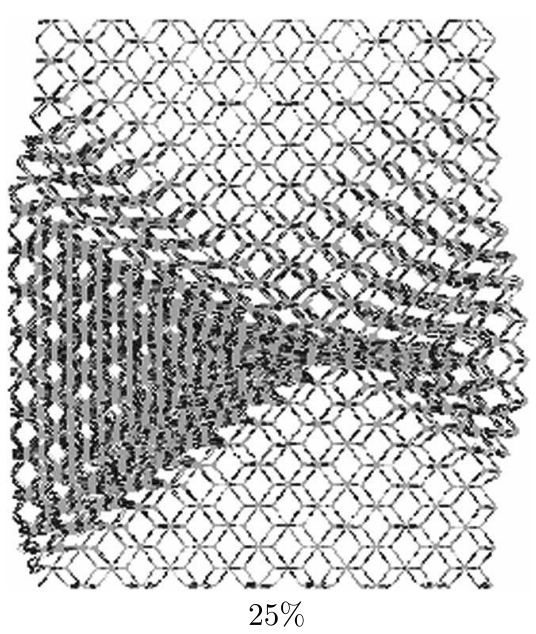

Fig. 5. Deformation of the irregular structure at different compressive strains

The stress-strain curves of the regular and irregular FE models of test sample 1 (Ti 120-500) are plotted and compared to each other in Fig. 6. Introduction of irregularity in the cross-section of the struts decreased both the yielding stress and densification strain. Both the values obtained from the irregular FE structure were closer to the experimental results (Table 3). Moreover, the stress-strain curve of the irregular structure was much smoother in the two points of yielding and densification (Fig. 6) which was in accordance to the observations in the stress-strain plots obtained from the experimental tests. Considering irregularity in the structure also decreased the elastic modulus by about 30\% (Fig. 6). The post-densification modulus was more influenced by considering the irregularities and was decreased by more than $50 \%$ (Fig. 6). 


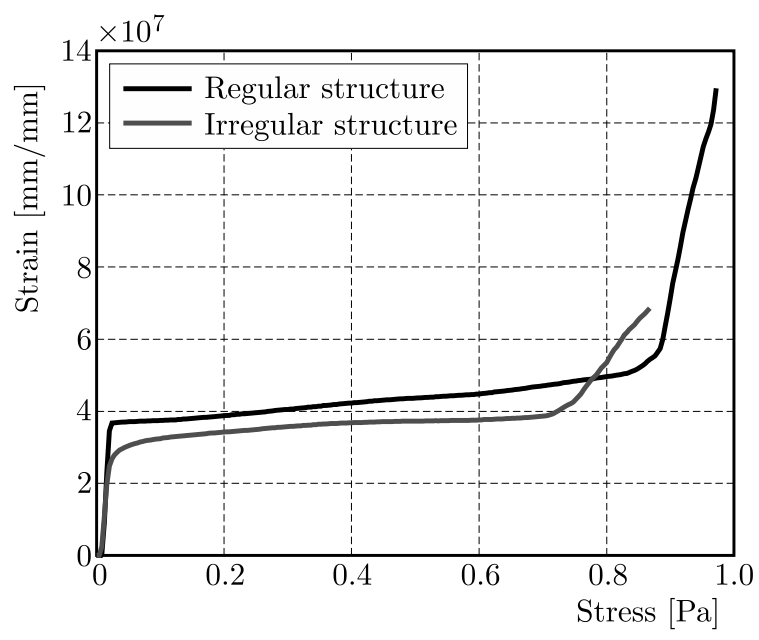

Fig. 6. Comparison of the stress-strain curves of the regular and irregular structures

Table 3. Comparison of plateau stress and densification strain of the numerical model and experimental tests of the four considered porous structures

\begin{tabular}{|l|c|c|c|c|}
\hline & $\begin{array}{c}\text { Sample 1 } \\
\text { Ti 120-500 }\end{array}$ & $\begin{array}{c}\text { Sample 2 } \\
\text { Ti 170-450 }\end{array}$ & $\begin{array}{c}\text { Sample 3 } \\
\text { Ti 170-500 }\end{array}$ & $\begin{array}{c}\text { Sample 4 } \\
\text { Ti 230-500 }\end{array}$ \\
\hline \hline$\varepsilon_{d}$ (FEM) & 0.71 & 0.75 & 0.76 & 0.65 \\
\hline$\varepsilon_{d}$ (Experiment) & 0.61 & 0.76 & 0.70 & 0.56 \\
\hline$\sigma_{p}$ (FEM) & $20 \mathrm{MPa}$ & $85 \mathrm{MPa}$ & $48 \mathrm{MPa}$ & $110 \mathrm{MPa}$ \\
\hline$\sigma_{p}$ (Experiment) & $15.8 \mathrm{MPa}$ & $67.8 \mathrm{MPa}$ & $34.8 \mathrm{MPa}$ & $91.8 \mathrm{MPa}$ \\
\hline
\end{tabular}

The elastic moduli obtained from theory (Babaee et al., 2012), our FEM code, and experimental tests (Amin Yavari et al., 2013) are compared in Fig. 7. As it can be seen, the numerical values are lower than the theoretical values due to the fact that variations in the cross-section areas have been considered in the FE model. However, the numerical elastic modulus is higher than that in the experimental tests, since the irregularities of diameter along each strut length has not been considered.

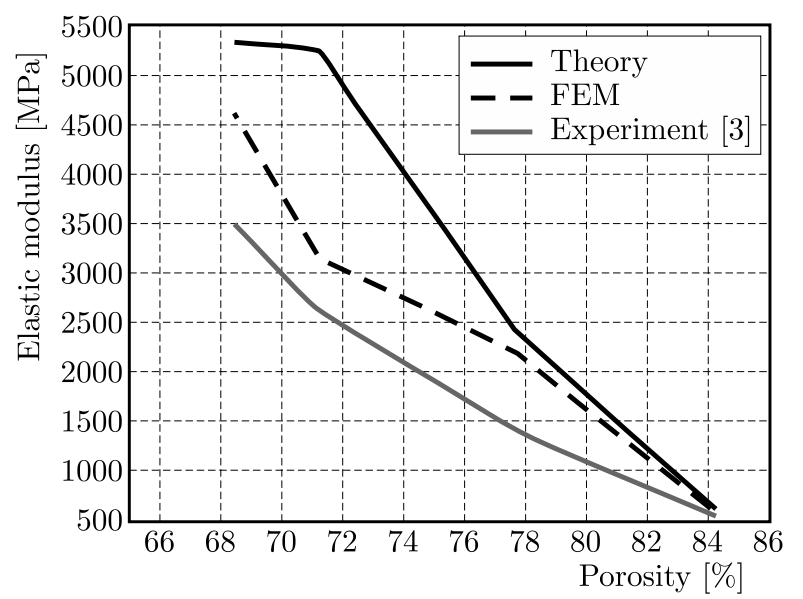

Fig. 7. Comparison of elastic moduli obtained from theory, our FEM code and experimental tests (Amin Yavari et al., 2013)

Using the obtained stress-strain curves (Fig. 8) from explicit simulations of the four cases (Table 1), the following values were calculated: elastic modulus $E$, strain at the beginning of the plateau region $\varepsilon_{p}$, densification strain $\varepsilon_{D}$, plateau stress $\sigma_{p}$, and the plateau end stress $\sigma_{D}$. 
As obvious in Fig. 9 and Table 3, the experimental and numerical results were relatively in good accordance. Both the plateau stress and densification strain of the FEM solution were larger than the experimental values (Fig. 9). This can be attributed to the lower amount of damage considered in the $\mathrm{FE}$ models procedure compared to the real irregularities present in the manufactured samples, as the irregularities along the strut length had not been considered in the FE models.

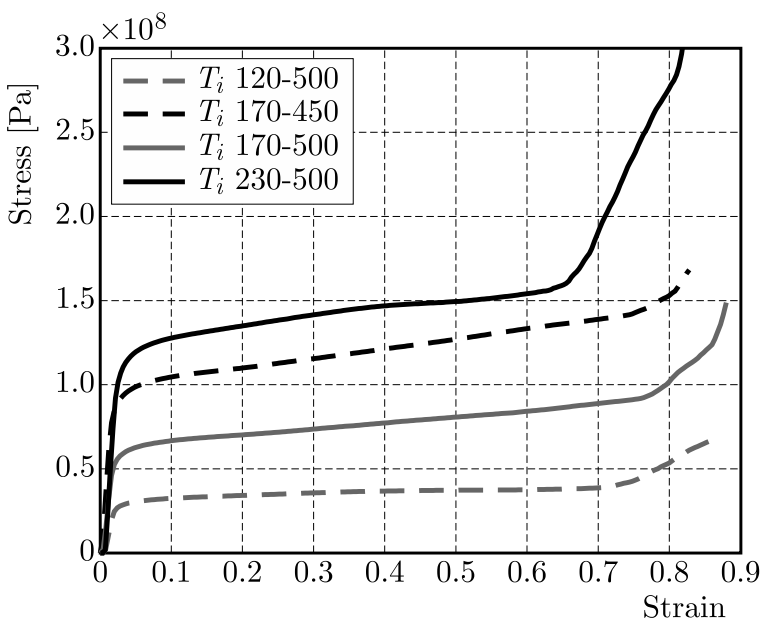

Fig. 8. Stress-strain curves for the four irregular samples
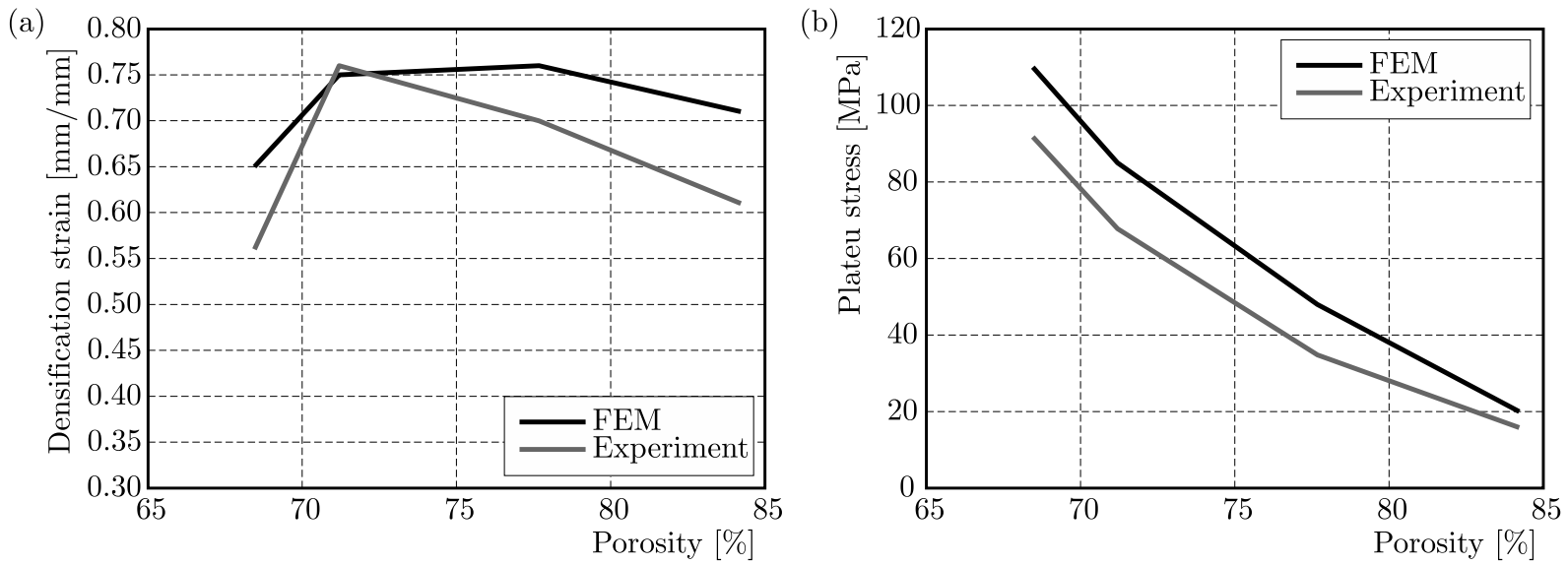

Fig. 9. Variation of (a) densification strain, and (b) plateau stress with respect to porosity

Both the numerical and experimental results show a decrease in the plateau stress by increasing the porosity (Fig. 9b). However, by increasing the porosity, the densification strain of the structure is initially increased and then decreases. This behaviour is observed in both the numerical and experimental curves (Fig. 9a). The maximum densification strain occurs for the relative density of about $70 \%$ (Fig. 9a).

\section{Discussions}

\subsection{Computational time}

In this study, the yielding behavior of open-cell foams was modeled using an explicit finite element code. In explicit finite element simulations, the structure is deformed through numerous time steps with very short durations. The time step is determined through the relationship 
$\Delta t=l / c$, where $l$ is the smallest element dimension throughout the entire model and $c$ is the speed of sound in the material. For yielding simulation, the calculated time step $\Delta t$ is usually very small (less than a microsecond). On the other hand, the yielding procedure is a quasi-static phenomenon which can take several minutes to be completed. This is why solving the yielding deformation using the explicit finite element method can lead to computational times larger than several days. The mass scaling was shown to be a very effective way of decreasing the solution time without having a significant effect on the obtained mechanical properties.

\subsection{Effect of irregularities}

$45^{\circ}$ deformation bands existed for both the regular and irregular lattice structures in postyielding regime (Figs. 4 and 5, see also (Silva and Gibson, 1997; Alkhader and Vural, 2008; Demiray et al., 2009). By applying the irregularity in cross-section areas of the struts, this $45^{\circ}$ deformation bands became vaguer (Fig. 5). This is because in the irregular structure, the struts start to fail sooner than they would fail in the regular structure. When the initial struts start to fail and, as a result, they are removed from the analysis, the load applied to their neighbor struts start to increase, and that makes the failure pattern grow around the initially failed struts as nucleuses. The results obtained from static and yielding simulations demonstrated that considering the irregularities has a significant effect on the response of the structure. In all the cases, considering the irregularities weakened the structure. Applying the irregularity in different cases decreased the plateau stress by $30-50 \%$ and the elastic modulus by $10-20 \%$. A similar decrease in the elastic modulus was reported by Campoli et al. (2013).

\subsection{Advantages of micro-mechanical modeling}

Several material models have been proposed in different studies for modeling the post-yielding behavior of foams in macro-mechanical analyses. For example, in LS-DYNA package, the material models MAT_LOW_DENSITY_FOAM, MAT_CRUSHABLE_FOAM, MAT_MODIFIED_CRUSHABLE_FOAM, MAT_SOIL_AND_FOAM, MAT_BLATZ-KO_FOAM, MAT_FU_CHANG, MAT_PITZER_CRUSHABLE_FOAM, MAT_DESHPANDE_FLECK_ FOAM, etc. are available for the modeling the behavior of the foam. Each of the abovementioned material models require several material constants to be determined through mechanical tests before being applicable in finite element simulations. For each foam type, the complex and time-consuming procedure of experimental tests for finding the constants must be repeated.

The micro-mechanical modeling of an open-cell foam has the advantage that the material constants of the foam structure do not have to be found through mechanical tests. In fact, in micro-mechanical models, the necessary material properties are those of the bulk material from which the foam is made of such as elastic modulus $E_{s}$, Poisson's ratio $\nu_{s}$, tangent modulus $T_{s}$, and yield stress $\sigma_{y_{s}}$.

\section{Conclusions}

In this paper, structures manufactured using the SLM technique and tested by Amin Yavari et al. (2013) were numerically modeled using ANSYS and LS-DYNA finite element codes for investigating the elastic and post-yielding behavior of the lattice structure, respectively. Implementing a micro-mechanical approach to the numerical modeling of the yielding behavior of open-cell porous materials is the main contribution of this work. One of the advantages of the micro-mechanical modeling of an open-cell structure is that, in contrast to the macro-mechanical finite element modeling, it is not necessary to obtain several material constants for different foam 
material models through heavy experimental tests. Regular and irregular lattice structures were created. After crushing the FE structure under compression, $45^{\circ}$ failure patterns were observed in both the regular and irregular structures, but more obvious in the regular structure. The failure patterns in the regular and irregular structures looked like sideways V and X signs, respectively. The results also showed that considering the irregularity in the cross-section area of different struts in the rhombic dodecahedron lattice structure decreases both the yielding stress and densification strain to values close to the experimental data. Moreover, compared to the regular FE structure, the stress-strain curve of the irregular FE structure was much smoother in the two points of yielding and densification, which was also observable in many experimental plots. Considering the irregularity in the structure also decreased the elastic modulus of the lattice structure by about 20-30\%. The post-densification modulus was more influenced by irregularity as it was decreased by more than $50 \%$. In summary, it is demonstrated that using beam elements with variable cross-sections for constructing open-cell porous biomaterials can give numerical results sufficiently close to the experimental data.

\section{References}

1. Ahmadi S., Campoli G., Amin Yavari S., Sajadi B., Wauthlé R., Schrooten J., Weinans H., ZADPOOR A.A., 2014, Mechanical behavior of regular open-cell porous biomaterials made of diamond lattice unit cells, Journal of the Mechanical Behavior of Biomedical Materials, 34, 106-115

2. Alkhader M., Vural M., 2008, Mechanical response of cellular solids: role of cellular topology and microstructural irregularity, International Journal of Engineering Science, 46, 10, 1035-1051 sufficiently

3. Amin Yavari S., Wauthlé R., Van der Stok J., Riemslag A., Janssen M., Mulier M., Kruth J.-P., Schrooten J., Weinans H., Zadpoor A.A., 2013, Fatigue behavior of porous biomaterials manufactured using selective laser melting, Materials Science and Engineering: C, 33, $8,4849-4858$

4. Babaee S., Jahromi B.H., Ajdari A., Nayeb-Hashemi H., Vaziri A., 2012, Mechanical properties of open-cell rhombic dodecahedron cellular structures, Acta Materialia, 60, 6, 2873-2885

5. Bitsche R., Daxner T., Böhm H.J., 2005, Space-Filling Polyhedra as Mechanical Models for Solidified Dry Foams, Technische Universität Wien

6. Borleffs M., 2012, Finite Element Modeling to Predict Bulk Mechanical Properties of 3D Printed Metal Foams, TU Delft, Delft University of Technology

7. Bram M., Stiller C., Buchkremer H.P., Stöver D., Baur H., 2000, High-porosity titanium, stainless steel, and superalloy parts, Advanced Engineering Materials, 2, 4, 196-199

8. Buffel B., Desplentere F., Bracke K., Verpoest I., 2014, Modelling open cell-foams based on the Weaire-Phelan unit cell with a minimal surface energy approach International Journal of Solids and Structures, 51, 19, 3461-3470

9. Campoli G., Borleffs M., Amin Yavari S., Wauthle R., Weinans H., Zadpoor A.A., 2013, Mechanical properties of open-cell metallic biomaterials manufactured using additive manufacturing, Materials and Design, 49, 957-965

10. Chen L.-J., Ting L., Li Y.-M., HaO H., Hu Y.-H., 2009, Porous titanium implants fabricated by metal injection molding, Transactions of Nonferrous Metals Society of China, 19, 5, 1174-1179

11. Demiray S., Becker W., Hohe J., 2009, Investigation of the fatigue behavior of open cell foams by a micromechanical 3-D model, Materials Science and Engineering: A, 504, 1, 141-149

12. Gibson L.J., Ashby M.F., 1997, Cellular Solids: Structure and Properties, Cambridge University Press 
13. Head W.C., Bauk D.J., Emerson R.H. JR, 1995, Titanium as the material of choice for cementless femoral components in total hip arthroplasty, Clinical Orthopaedics and Related Research, 311, 85-90

14. Hedayati R., Hosseini-Toudeshiy H., Sadighi M., Mohammadi-Aghdam M., Zadpoor A.A., 2016a, Computational prediction of the fatigue behavior of additively manufactured porous metallic biomaterials, International Journal of Fatigue, 84, 67-79

15. Hedayati R., Sadighi M., Mohammadi-Aghdam M., Zadpoor A.A., 2016b, Effect of mass multiple counting on the elastic properties of open-cell regular porous biomaterials, Materials and Design, 89, 9-20

16. Hedayati R., Sadighi M., Mohammadi-Aghdam M., Zadpoor A.A., 2016c, Mechanical behavior of additively manufactured porous biomaterials made from truncated cuboctahedron unit cells, International Journal of Mechanical Sciences, 106, 19-38

17. Hedayati R., Sadighi M., Mohammadi-Aghdam M., Zadpoor A.A., 2016d, Mechanical properties of regular porous biomaterials made from truncated cube repeating unit cells: analytical solutions and computational models, Materials Science and Engineering: C, 60, 163-183

18. Hedayati R., Sadighi M., Mohammadi-Aghdam M., Zadpoor A.A., 2016e, Mechanics of additively manufactured porous biomaterials based on the rhombicuboctahedron unit cell, Journal of the Mechanical Behavior of Biomedical Materials, 53, 272-294

19. Heinl P., Müller L., Körner C., Singer R.F., Müller F.A., 2008, Cellular Ti-6Al-4V structures with interconnected macro porosity for bone implants fabricated by selective electron beam melting, Acta Biomaterialia, 4, 5, 1536-1544

20. Karageorgiou V., Kaplan D., 2005, Porosity of 3D biomaterial scaffolds and osteogenesis, Biomaterials, 26, 27, 5474-5491

21. KrayniK A.M., Reinelt D.A., 1996, Linear elastic behavior of dry soap foams, Journal of Colloid and interface Science, 181, 2, 511-520

22. Kwok P.J., Oppenheimer S.M., Dunand D.C., 2008, Porous titanium by electro-chemical dissolution of steel space-holders, Advanced Engineering Materials, 10, 9, 820-825

23. Lu J., Flautre B., Anselme K., Hardouin P., Gallur A., Descamps M., Thierry B., 1999, Role of interconnections in porous bioceramics on bone recolonization in vitro and in vivo, Journal of Materials Science: Materials in Medicine, 10, 2, 111-120

24. Luxner M.H., Woesz A., Stampfl J., Fratzl P., Pettermann H.E., 2009, A finite element study on the effects of disorder in cellular structures, Acta Biomaterialia, 5, 1, 381-390

25. Mullen L., Stamp R.C., Brooks W.K., Jones E., Sutcliffe C.J., 2009, Selective laser melting: a regular unit cell approach for the manufacture of porous, titanium, bone in-growth constructs, suitable for orthopedic applications, Journal of Biomedical Materials Research, Part B: Applied Biomaterials, 89, 2, 325-334

26. Parthasarathy J., Starly B., Raman S., Christensen A., 2010, Mechanical evaluation of porous titanium (Ti6Al4V) structures with electron beam melting (EBM), Journal of the Mechanical Behavior of Biomedical Materials, 3, 3, 249-259

27. Ptochos E., Labeas G., 2012a, Elastic modulus and Poisson's ratio determination of microlattice cellular structures by analytical, numerical and homogenisation methods, Journal of Sandwich Structures and Materials, p. 1099636212444285

28. Ptochos E., Labeas G., 2012b, Shear modulus determination of cuboid metallic open-lattice cellular structures by analytical, numerical and homogenisation methods, Strain, 48, 5, 415-429

29. Ryan G., Pandit A., Apatsidis D.P., 2006, Fabrication methods of porous metals for use in orthopaedic applications, Biomaterials, 27, 13, 2651-2670 
30. Shulmeister V., Van der Burg M., Van der Giessen E., Marissen R., 1988, A numerical study of large deformations of low-density elastomeric open-cell foams, Mechanics of Materials, 30, $2,125-140$

31. Silva M.J., Gibson L.J., 1997, The effects of non-periodic microstructure and defects on the compressive strength of two-dimensional cellular solids, International Journal of Mechanical Sciences, 39, 5, 549-563

32. Warren W., Kraynik A., 1997, Linear elastic behavior of a low-density Kelvin foam with open cells, Journal of Applied Mechanics, 64, 4, 787-794

33. Zheng X., Lee H., Weisgraber T.H., Shusteff M., DeOtte J., Duoss E.B., Kuntz J.D., Biener M.M., GE Q., JaCKson J.A., 2014, Ultralight, ultrastiff mechanical metamaterials, Science, 344, 6190, 1373-1377

Manuscript received October 29, 2014; accepted for print November 23, 2015 